\title{
A SIMPLE IDIOTYPIC NETWORK MODEL WITH COMPLEX DYNAMICS
}

\author{
R. J. De Boer ${ }^{1}$, I. G. Kevrekidis ${ }^{2}$, and A. S. Perelson ${ }^{1}$ \\ 1Theoretical Division, Los Alamos National Laboratory, Los Alamos, NM 87545 \\ ${ }^{2}$ Department of Chemical Engineering, Princeton University, Princeton, NJ 08544
}

\begin{abstract}
According to the network theory of the immune response, the various $B$ and $T$ lymphocyte clones that comprise the immune system respond to antigen in a coordinated way due to idiotypic interactions. Many models of jdiotypic interactions have ignored the fact that cells communicate by secreting soluble molecules, and that the chemical reactions between these soluble molecules and cell surface receptors control key features of the immune response. Here we develop and analyze a set of simple idiotypic network models in which cells and molecules are distinguished, and which incorporate realistic chemical interactions among the various cellular and molecular species. Our first, most complete model involves a set of eleven ordinary differential equations. Using two time scale analysis we find that on a long time scale the chemical reactions come to equilibrium, and we need only consider a set of six differential equations with algebraic constraints. We then show that a reduced four differential equation model captures most of the dynamic features of the full model. Using numerical bifurcation techniques we study the steady state and dynamic behavior of the reduced model for a range of realistic parameter values. Our models exhibit multiple steady states, limit cycle oscillations and chaotic behavior. Comparison with experimental data collected in vivo show that these dynamical features are characteristic of certain immune phenomeria.
\end{abstract}

\section{KEYWORDS}

Immune system, idiotypic networks, numerical bifurcation, chaos

\section{INTRODUCTION}

Dynamical models in immunology have generally dealt with describing the overall behavior of the immune system. In the case of the humoral immune response this has lead to models which predict the time courac of incrense in serum antibody level after antigen injection, and the more rapid higher amplitude increase seen after a seconcl injection with the same antigen. Modeling these characteristics of the primary and secondary immune responses has been the testing ground for immunological models for the past two decades. Recently, due to the development of monoclonal anti-idiotypic antibodies, experimental information has become available (Lundkvist et al., 1989) on the dynamical changes in a few clones secreting antibody with the same idiotype in circumstances where no antigen has been injected. In these experiments aperiodic, apparently chaotic behavior is observech, with fluctuations having amplitudes much smaller than those characteristic of the immune response to antigen. Here we develop a dynamical model, which we believe captures many of the important events in $B$ cell stimulation, and which exhibits the type of complex dynamics recently observed at the level of single idiotypes. In this model, the immune system is modeled as a chemostat, with inflow terms corresponding to the generation of $B$ cells from the bone marrow, and outflow corresponding to cell death and degradation of soluble molecules.

Models of idiotypic networks have recently been reviewed (Perelson, 1989). The basic idea behind idiotypic network theory is that the immune system can respond to novel determinants (idiotopes) on its own molecules. Thus, the immune system can (and, in fact, does) make antibodies that recognize other antibodies or cell surface receptors within the same animal. Jerne (1974) proposed that such idiotypic interactions played a regulatory role in the immune system. Although $T$ cells are also important in immune regulation, here we develop a class of models that rely only on idiotypic interactions among B cells for their regulation. These models can be viewed as providing a limiting example of what can be accomplished without specific $\mathbf{T}$ cell intervention.

\section{A MODEL OF CHEMICAL INTERACTIONS BETWEEN ANTIBODIES AND B-CELLS}

Antibody is produced by $B$ lymphocytes, which have on their surface immunoglobulin molecules as receptors. These immunoglobulin receptors are essentially identical to the antibody molecule the $B$ cell will secrete, with the exception that the receptor contains a hydrophobic tail that inserts into the $B$ cell membrane. When the 
receptors on a B cell are cross-linked, the B cell under appropriate circumstances can become "activated". An activated $B$ cell begins proliferating and differentiates into an antibody secreting state. The chemistry of receptor cross-linking has been well studied (Perelson, 1984) and forms the basis of the following Antibody B-cell Chemistry (ABC) model.

Consider a system composed of $\mathbf{B}$ cell subpopulations, $B_{i}, i=1, \ldots, n$. Each subpopulation when stimulated grows according to

$$
\frac{d B_{i}}{d t}=m+p f\left(h_{i}\right) B_{i}-d_{B} B_{i}
$$

where $m$ is the rate of generation of new $B_{i}$ cells in the bone marrow and $d_{B}$ is their specific death rate. Stimulated B cells proliferate with rate constant $p$. The fraction of cells stimulated to grow, $f$, depends on the degree of receptor cross-linking, $h_{i}$, on cell $i$.

When stimulated, $B_{i}$ will secrete antibody, $A_{i}$, at rate $s_{i}(t)$. Because there is an induction period before antibody secretion, we assume

$$
\frac{d s_{i}}{d t}=k_{s}\left(s f\left(h_{i}\right)-s_{i}\right)
$$

where $s$ is the maximum rate of antibody secretion and $k_{s}$ determines the rate of induction. At times long compared with $1 / k_{s}$, the secretion rate is approximately $s f\left(h_{i}\right)$, i.e. depends upon the amount of receptor cross-linking.

Secreted antibody decays at rate $d_{A}$, and reacts chemically with other antibody (or antigen) in solution to form complexes, $C$, which are eliminated from the body at an enhanced rate $d_{C}>d_{A}$. Antibody also binds to and cross-links cell surface immunoglobulin. The major forms of secreted antibody are IgG and IgM. IgG is bivalent and IgM 10-valent. For simplicity, we assume all antibody is bivalent and thus can be in the states: free ( $A_{i}$ ), bound at one site to a receptor on cells $j\left(A_{i j}^{\prime}\right)$ or bound at both sites to receptors on cells $j\left(A_{i j}^{\prime \prime}\right)$.

Immunoglobulin receptors are all bivalent. We shall assume that the reactivity of one receptor site is independent of the state of the other site on the same receptor. With this equivalent site hypothesis we can ignore the fact that receptors are bivalent and deal only with the concentration of free receptor sites on cells $j, S_{j}$ (Perelson and DeLisi, 1980). The concentration of free receptor sites per cell is $S_{j} / B_{j}$. With these assumptions,

$$
\begin{gathered}
\frac{d A_{i}}{d t}=s_{i} B_{i}-d_{A} A_{i}-2 \sum_{j} k_{i j}^{+} A_{i} S_{j}+\sum_{j} k_{i j}^{-} A_{i j}^{\prime}-4 \sum_{j} k_{i j}^{c+} A_{i} A_{j}+\sum_{j} k_{i j}^{c-} C_{i j} \\
\frac{d A_{i j}^{\prime}}{d t}=2 k_{i j}^{+} A_{i} S_{j}-k_{i j}^{-} A_{i j}^{\prime}-k_{i j}^{x+} A_{i}^{\prime} S_{j} / B_{j}+2 k_{i j}^{x-} A_{i j}^{\prime \prime} \\
\frac{d A_{i j}^{\prime \prime}}{d t}=k_{i j}^{x+} A_{i}^{\prime} S_{j} / B_{j}-2 k_{i j}^{x-} A_{i j}^{\prime \prime} \\
\frac{d C_{i j}}{d t}=4 k_{i j}^{c+} A_{i} A_{j}-k_{i j}^{c-} C_{i j}-d_{C} C_{i j}
\end{gathered}
$$

where $k_{i j}^{+}, k_{i j}^{-}$are the rate constants for the binding of $A_{i}$ to a site on $B_{j}, k_{i j}^{x+}, k_{i j}^{x-}$ are the rate constants for $A_{i j}^{\prime}$ binding to a second receptor site on the same cell and forming a cross-link, and $k_{i j}^{c+}, k_{i j}^{c-}$ are the rate constants for the reaction of $A_{i}$ with $A_{j}$ in solution. (For simplicity, we have ignored the formation of larger solution phase antibody-antibody complexes.) The number of cross-links and free sites on subpopulation $j$ are given by,

$$
h_{j}=\sum_{i} A_{i j}^{\prime \prime}, \quad S_{j}=B_{j} R_{T}-\sum_{i} A_{i j}^{\prime}-2 \sum_{i} A_{i j}^{\prime \prime},
$$

where $R_{T}$ is the total number of receptor sites on a single cell, here assumed to be a constant. The total number of cross-links on a cell is at most $R_{T} / 2$. Thus, we choose as an activation function

$$
f\left(h_{i}\right)=\frac{2 h_{i}}{B_{i} R_{T}}
$$

We have studied the above system for $n=2$ and $k_{i i}^{+}=k_{i i}^{c+}=0, i=1,2$ (Perelson, 1989). For this case, in which $A_{1}$ binds only to $B_{2}$ and $A_{2}$ (and vice-versa), the model reduces to a set of 11 differential equations. Because the chemical reactions typically take place on a time scale of seconds to minutes, whereas changes in the $B$ cell population, the induction period for antibody secretion, and changes in the total antibody concentration take place on a time scale of hours to days, one can use the method of multiple scales to further simplify the model (Segel and Perelson, 1989). On the long time scale, the chemical reactions can all be assumed to be at equilibrium. The model then reduces to six differential equations (for $B_{1}, B_{2}, A_{1 T}, A_{2 T}, s_{1}$ and $s_{2}$ ) with a set of algebraic constraints, where $A_{i T}=A_{1}+A_{i}^{\prime}+A_{i}^{\prime \prime}+C$ is the total concentration of antibody $i$. A major feature is that the number of cross-links on cell 1 (2) is a bell-shaped function of $A_{2}\left(A_{1}\right)$ whose midpoint and width depend on the equilibrium constants $K_{12}$ and $K_{12}^{x}$, respectively, (Perelson and DeLisi, 1980). Thus at low concentrations 


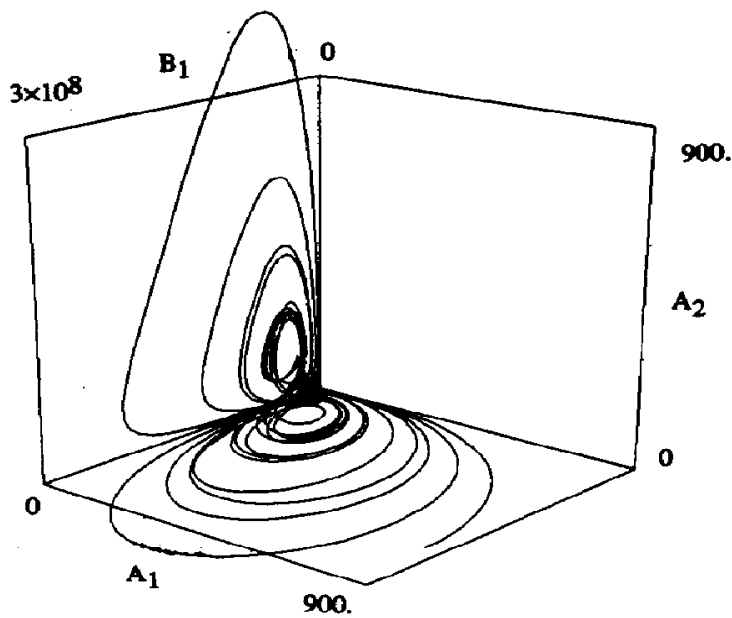

Fig. 1. Chaotic behavior in the ABC model in a phase space showing $B_{1}, A_{1}$, and $A_{2}$. The trajectory resembles the Lorenz attractor. Parameters: $m=2400$ cells d $\mathrm{d}^{-1}, p=1 \mathrm{~d}^{-1}, d_{B}=0.3 \mathrm{~d}^{-1}, d_{A}=0.1 \mathrm{~d}^{-1}, d_{C}=2$ $\mathrm{d}^{-1}, k_{s}=0.2 \mathrm{~d}^{-1}, s=1.44 \times 10^{-6} \mathrm{nmol}$ cell-1 $\mathrm{d}^{-1}, R_{T}=10^{5}$ receptor sites $/$ cell $=1.67 \times 10^{-10} \mathrm{nmol} / \mathrm{cell}, k_{i j}^{-}=k_{i j}^{x-}=k_{i j}^{c-}=0.1 \mathrm{~s}^{-1}, K_{i j}=$ $K_{i j}^{c}=10 \mathrm{~cm}^{3} \mathrm{nmol}^{-1}, K_{i j}^{x}=10^{11}(\mathrm{nmol} / \mathrm{cell})^{-1}$ (see Perelson, 1989).

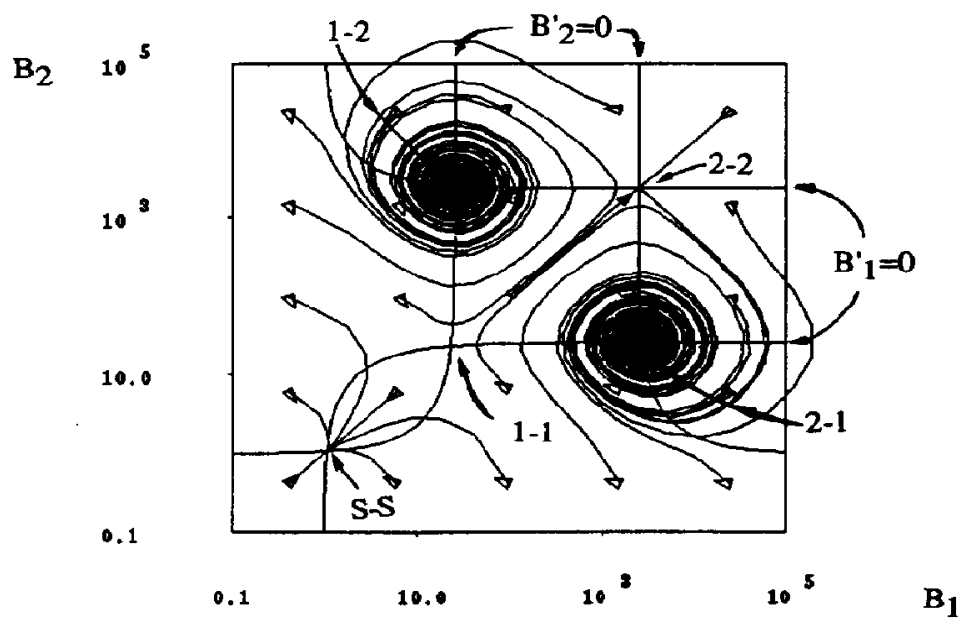

Fig. 2. The 2 dimensional $B$ model. Indicated are the $B_{1}$ and the $B_{2}$ null-clines, and a set of trajectories showing the stability of equilibria. The null-lines intersect at five equilibria which are marked by the abbreviations we use in the text. Trajectories and null-clines are drawn by GRIND (De Boer, $1983)$.

of $A_{2}, B_{1}$ is stimulated but at high concentrations it is suppressed. Using realistic parameter choices both the 11 and 6 equation versions of the model exhibit multiple steady states, limit cycle oscillations, and chaos (Perelson, 1989 and Fig. 1). The attractor shown in Fig. 1 resembles that seen in the Lorenz equations (Lorenz, 1963). To understand this behavjor in detail, we further simplify the model. 


\section{SIMPLIFIED MODELS}

One of the most striking results of this paper is that the behavior of the ABC model is very similar to that of a far simpler model which summarizes all chemistry into a simple bell-shaped function and which ignores the time delay involved in antibody secretion. This model considers $\mathbf{B}$ cells $\left(b_{i}\right)$, antibodies $\left(a_{i}\right)$, a bell-shaped activation function $\left(f\left(h_{i}\right)\right.$ ) which is determined by the symmetric affinity matrix $\mathbf{J}_{i j}$, and the field of each population ( $h_{i}$ ); it was derived previously (De Boer and Hogeweg, 1989a,b). We will refer to it as the "AB" model (for Antibody-B cell):

$$
\begin{gathered}
h_{i}=\sum_{j} \mathbf{J}_{i j} a_{j}, \quad f\left(h_{i}\right)=\frac{h_{i}}{\Theta_{1}+h_{i}} \frac{\Theta_{2}}{\Theta_{2}+h_{i}}, \\
\frac{d b_{i}}{d t}=m+p f\left(h_{i}\right) b_{i}-d_{B} b_{i}, \quad \frac{d a_{i}}{d t}=s b_{i} f\left(h_{i}\right)-d_{C} h_{i} a_{i}-d_{A} a_{i}
\end{gathered}
$$

We non-dimensionalize this model by setting a time scale: $\tau=t d_{B}$. Since $b_{i}=m / d_{B}$ is a typical "virgin" $B$ cell population (see below), we let $B_{i}=b_{i} d_{B} / m$. Further let $\alpha$ be a typical antibody concentration, and let $A_{i}=a_{i} / \alpha$. Then the model simplifies into:

$$
\begin{gathered}
h_{i}=\sum_{j} \mathbf{J}_{i j} A_{j}, \quad f\left(h_{i}\right)=\frac{h_{i}}{\theta_{1}+h_{i}} \frac{\theta_{2}}{\theta_{2}+h_{i}}, \\
\frac{d B_{i}}{d \tau}=1+B_{i}\left(r f\left(h_{i}\right)-1\right), \quad \frac{d A_{i}}{d \tau}=B_{i} f\left(h_{i}\right)-A_{i}\left(\mu h_{i}+\delta\right),
\end{gathered}
$$

where $\alpha=m s / d_{B}^{2}, r=p / d_{B}, \mu=\alpha d_{C} / d_{B}, \delta=d_{A} / d_{B}, \theta_{1}=\Theta_{1} / \alpha$ and $\theta_{2}=\Theta_{2} / \alpha$. The physical interpretation of the parameters is as follows. $r$ is the ratio of the maximum growth rate to the rate of cell death. $\delta$ is the ratio of the rates of decay of antibodies and $\mathrm{B}$ cells. $\mu$ is the ratio of the rate at which antibodies in complexes are removed to $B$ cell death rate.

Because the AB model is so much simpler than the ABC model, we will present resuits of a bifurcation analysis of the AB model in the remainder of this paper. In order to interpret the steady states present in this model it is useful to consider an even simpler version in which the distinction between cells and antibodies is ignored. We refer to this model as the "B" model (for B cell). It is obtained by omitting the antibody equations and replacing Eq. 7 by: $h_{i}=\sum_{j} \mathbf{J}_{i j} B_{j}$. For $n=2$, this $\mathrm{B}$ model consists of two differential equations; its null-clines are depicted in Fig. 2 (for $J_{11}=\mathbf{J}_{22}=0$, and $\mathbf{J}_{12}=\mathbf{J}_{21}=1$ ). Below we described the correspondence in steady states between the two simplified models.

The parameters used here are based on the following considerations, discussed in more detail in De Boer and Hogeweg ( $1989 \mathrm{~b})$ : the bone marrow produces one cell per clone per day $\left(m=1 \mathrm{~d}^{-1}\right)$; populations expand about 100 -fold $\left(\Theta_{1}=10^{2}, \Theta_{2}=10^{4}\right)$; activated cells divide every 16 hours $\left(p=1 \mathrm{~d}^{-1}\right)$; $\mathrm{B}$ cells have a life-time of about $2 \mathrm{~d}\left(d_{B}=0.5 \mathrm{~d}^{-1}\right)$; antibodies have a life-time of about $20 \mathrm{~d}\left(d_{A}=0.05 \mathrm{~d}^{-1}\right)$; the secretion rate is normalized: a fully stimulated $B$ cell produces one unit of antibody per day ( $s=1$ unit $d^{-1}$ ); antibody complexes are removed at a rate $d_{C}=10^{-3} \mathrm{~d}^{-1}$ unit -1 . With these parameter, $r=2, \theta_{1}=25, \theta_{2}=2500, \mu=8 \times 10^{-3}$, and $\delta=0.1$. In this paper our two basic bifurcation parameters are $\mu$, for which reasonable values range between $0 \leq \mu \leq 0.1$, and $\delta$, which we have studied for $0.01 \leq \delta \leq 1$.

\section{BIFURCATION RESULTS}

Five of the six steady states that are present in the AB model for $n=2$ are also present in the 2 -dimensional B model (Fig. 2). At low population levels one finds a "virgin state" in which $f\left(h_{i}\right) \simeq 0$ and there is a balance between the source of new cells and cell death. This state is present whenever $\theta_{1}>>1$ (De Boer and Hogeweg, $1989 \mathrm{a}$ ). We refer to it as the "S-S" state (for small-small) since both $B_{1}$ and $B_{2}$ are small (in fact, $B_{i} \simeq 1$ ). The four other states, which we Iefer to as "1-1", "1-2", "2-1", and "2-2", have $B_{1}$ and $B_{2}$ at approximately $\theta_{1}$ or $\theta_{2}$. State "1-2" corresponds to $B_{1} \simeq \theta_{1}$ and $B_{2} \simeq \theta_{2}$, etc. (In the AB model these same five steady states appear but with $A_{i}$ rather than $B_{i}$ approximately $\theta_{1}$ or $\theta_{2}$.) At the thresholds, $f\left(h_{i}\right) \simeq 0.5$. Thus, for $r=2$, $B_{i}\left(r f\left(h_{i}\right)-1\right) \simeq 0$. Since the source term is small, large $B_{i}$ populations attain an equilibrium. The symmetric states, 1-1 and 2-2, are unstable and presumably would not be observed in the immune system. We found the two asymmetric steady states, $1-2$ and $2-1$, to be stable over a large interval of realistic parameter values. They have been called immune states and may be associated with immunological memory (De Boer and Hogeweg, 1989b; Weisbuch et al., 1990).

In the $A B$ model, one virgin state is always present at $B_{1}=B_{2}=1$ and $A_{1}=A_{2}=0$. Since antibody levels are zero, this state is devoid of any $B$ cell stimulation. We refer to it as the " $0-0$ " state. Thus two virgin states exist (S-S and 0-0); however, as we show below, for each parameter setting only one of them is stable. We have searched for bifurcations of the six steady states in the AB model. The bifurcation calculations were performed using AUTO (Doedel, 1981); the phase-portraits were obtained with GRIND (De Boer,1983) and SCIGMA (Kevrekidis and Jolly, 1987). Varying the parameters $\mu$ and $\delta$ along the lines $\delta=0.1$ and $\mu=0.008$ respectively, we found a (subcritical) Hopf-bifurcation at $\mu=0.0507, \delta=0.1$ and a branch point between the two virgin states at $\delta=0.04, \mu=0.008$. 
The Hopf-bifurcation is further explored in Fig. 3. This two-parameter bifurcation curve shows the location of the Hopf-bifurcation as a function of $\mu$ and $\delta$. The inset is a blow-up of the S-shaped region at low $\mu$ values. We conclude from Fig. 3 that the Hopf-bifurcation only exists for reasonable values of $\mu$ if $\delta$ is sufficiently small (i.e. $\delta<0.2$ ). This confirms our previous result (De Boer and Hogeweg, 1989b) that the life-time of antibodies and cells has to differ significantly to obtain oscillations. Fig. 4 shows a one-parameter continuation of $\mu$ in the neighborhood of the Hopf bifurcation. At high $\mu$ values the immune states are stable (only the 2-1 state, $A_{1} \simeq \theta_{2}, A_{2} \simeq \theta_{1}$, is shown in Fig. 4). As $\mu$ increases toward 0.0507 , the initially unstable immune states become stable at the Hopf bifurcation, each giving rise to a subcritical unstable limit cycle. The insets in Fig. 4 a indicate the eigenvalue or Floquet multiplier structure. Fig. 4b shows the shape of the unstable limit cycle in the $B_{1}$ vs. $A_{1}$ plane. For increasing values of $\mu$ the amplitude of the limit cycle increases; this indicates that the basin of attraction of the stable immune state increases. The period of the unstable limit cycle remains fairly constant (not shown).

We explore the stability of the virgin states in Fig. 5 for $\mu=0.008$. The $0-0$ state is stable and the S-S state is unstable for $\delta>0.04$. At $\delta=0.04$ there is an exchange of stability and the S-S state becomes stable and $0-0$ unstable. At low values of $\delta$ antibodies are long-lived; the 0-0 state is unstable in this region because $B$ cells that are marginally stimulated are able to maintain a low concentration of antibody. Conversely, if antibodies are relatively short-lived the $0-0$ state is the only stable virgin state. At sufficiently large values of $s$ the $S-S$ and 1-1 states disappear (see De Boer and Hogeweg, 1989a).

The symmetric states, 0-0, S-S, 1-1, and 2-2, are all located in the invariant subspace $B_{1}=B_{2}$ and $A_{1}=A_{2}$ (in Fig. 2 this corresponds to the diagonal $B_{1}=B_{2}$ ). The one-dimensional unstable manifold (i.e. the outset) of the 1-1 state lies in this invariant subspace. To the left in Fig. 6 (and Fig. 2) this outset approaches the virgin state, to the right it approaches the 2-2 state. The 2-2 state is stable on this invariant subspace. Its single unstable direction is normal to the invariant subspace. Fig. 6 shows a schematic of the connections between these states. Leaving the 2-2 state along its unstable manifold we approach the vicinity of the $1-1$ state in a spiral. For $\mu=0.041$ this spiral eventually turns and is attracted to the virgin state. For $\mu=0.0405$ this spiral, after leaving the 1-1 neighborhood approaches an apparent chaotic attractor. Between these two values of $\mu$ we expect the existence of a heteroclinic connection (i.e. a connection between different states) between 1-1 and 2.2 , (a global bifurcation), giving rise to long transients in the neighborhood of the 1-1 unstable state.

For certain values of $\mu$ the AB model displays chaotic behavior. If one compares the chaotic attractor of the ABC model (Fig. 1) with that of the AB model (not shown) one finds that they are remarkably similar. This shows that the AB model captures the essence of the behavior of the ABC model. The attractors of both models resemble that of the Lorenz equations. This similarity extends beyond visual resemblance. Indeed, the variation of the hehavior of the $A B$ model as $\mu$ varies, bears many common features with the variation of the dynamics of the Lorenz system with respect to the parameter $r$ (discussed, for example, by Sparrow, 1982). There exist several windows of periodic behavior interspersed within the chaotic regime. The limit cycles can either be symmetric (under the interchange of the subscripts 1 and 2) or asymmetric, arising in pairs by symmetry-breaking (pitchfork) bifurcations. The asymmetric cycles undergo a cascade of period doublings, eventually resulting in apparently chaotic dynamics. At various values of $\mu$ we have observed "noisy-periodicity" (e.g. $\mu=0.0056)$ and intermittent behavior (e.g. $\mu=0.00478$ ).

Around the Hopf-bifurcation at $\mu=0.0507$ and $\delta=0.1$ a region of hysteresis arises. Below the Hopf $(\mu<0.0507)$, the immune states (1-2 and 2-1) are unstable. The system has a stable virgin state; outside its basin of attraction the system exhibits large amplitude, chaotic, or, in parameter windows, periodic behavior. Above the Hopf $(\mu>0.0507)$, the two immune states are stable and each is surrounded by a large amplitude unstable limit cycle of the sactlle type. These unstable limit cycles, along with their stable manifolds, define the basins of a.t.traction of the immune states. A stable virgin state is also present. For $0.0507 \leq \mu<0.056$, one finds outside the basins of attraction of the stable immune states the "butterfly" attractor (Fig. 1). In this parameter region hysteresis is observed between the stable chaotic attractor and the stable immune state. The chaotic attractor does not appear to exist above $\mu \simeq 0.056$. Our numerical calculations strongly indicate that its death at $\mu \simeq 0.056$ is associated with a global interaction of the stable and unstable manifolds of the two asymmetric limit cycles (Fig. 4b). This is supported by the observation of long "chaotic" transients, with unpredictable winding patterns around the two limit cycles, which, for $\mu>0.056$, eventually settle into one of the immune states.

Lundkvist et al. (1989), measured in mice the oscillatory patterns in the concentrations of two complementary antibodies, e.g. $A_{1}$ and $A_{2}$. They showed that following the injection of one of the two antibodies, these oscillatory patterns were absent for several weeks. In Fig. 7 we describe a similar experiment in our $A B$ model (for $\mu=0.008, \delta=0.1$ ). Following $100 \mathrm{~d}$ of chaotic cycles we introduce $10^{5}$ units of $A_{2}$. This rapidly removes nearly all $A_{1}$ by complex formation. As a consequence, $B_{2}$ is no longer stimulated to proliferate or to produce $A_{2}$. $A_{2}$ decays by normal antibody turnover. Oscillations are absent until $A_{2}$ falls below $\theta_{2}$ and stimulates $B_{1}$ to produce $A_{1}$. After about $50 \mathrm{~d}$ the behavior is chaotic again; the time until chaos returns depends on the dose of $A_{2}$ and the rate of antibody turnover, $d_{A}$. Fig. 7 is in close correspondence with the empirically measured data. There is one discrepancy, however, in that in the experiment one does not observe high concentrations of $A_{2}$ during the quiescent period. The experimentalists believe that the dose of injected antibody, when diluted in the volume of the animal, only generates a small perturbation of the natural antibody concentration. 


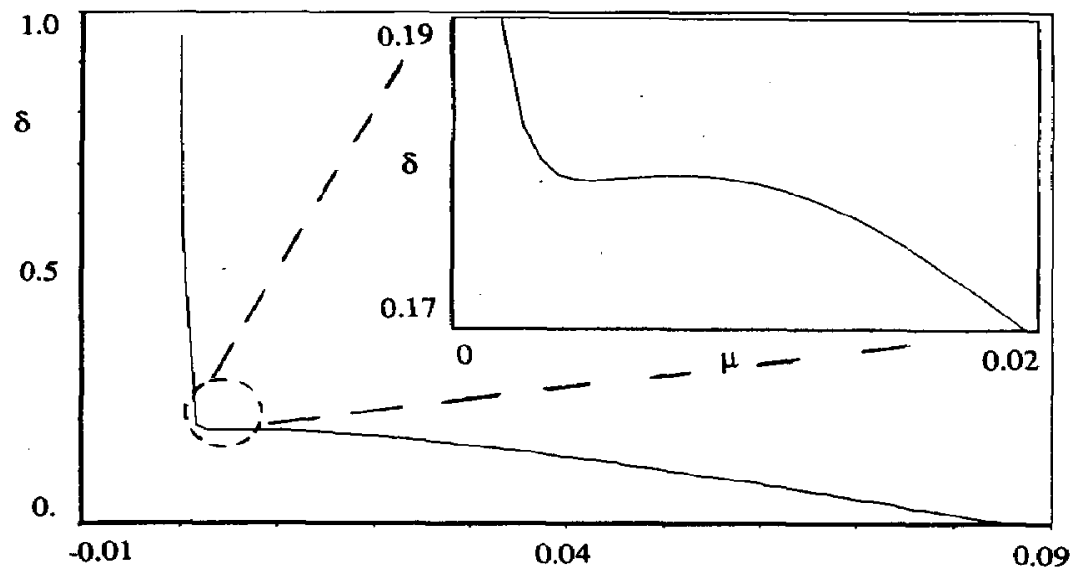

Fig. 3. The location of the Hopf-bifurcation of the AB model as a function of $\mu$ and $\delta$. The inset shows the S-shaped region at low values of $\mu$.
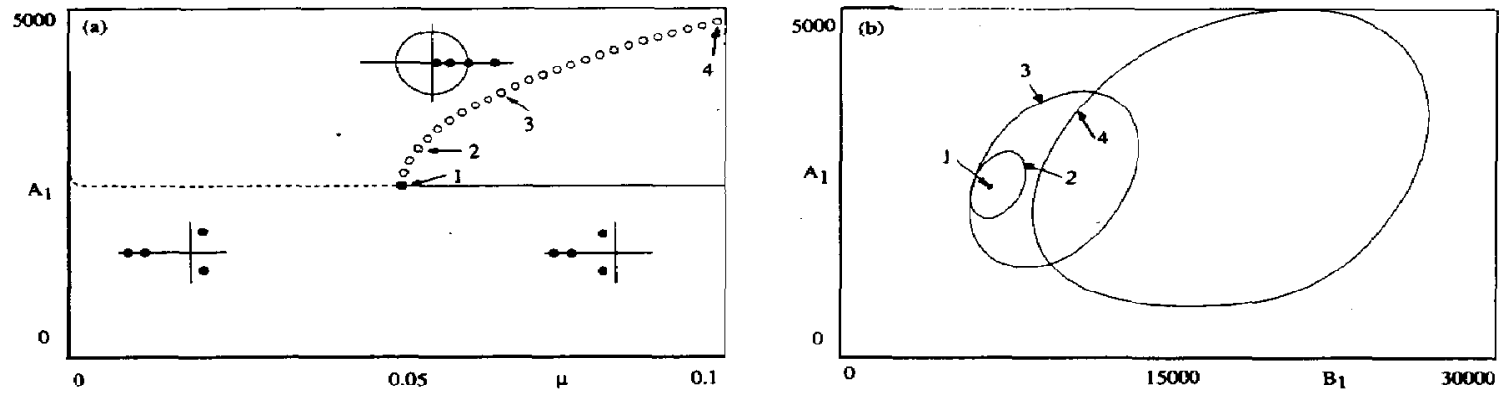

Fig. 4. A one-parameter continuation of $\mu$ around the Hopf-bifurcation of the AB model. (a) The changc in stability of the 1-2 state; - - - unstable equilibria, - stable equilibria, o o o unstable limit cycles. The diagrams give the eigenvalue structure. (b) The unstable limit cycles in the $B_{1}$ vs. $A_{1}$ plane. The numbers correspond to those in (a).

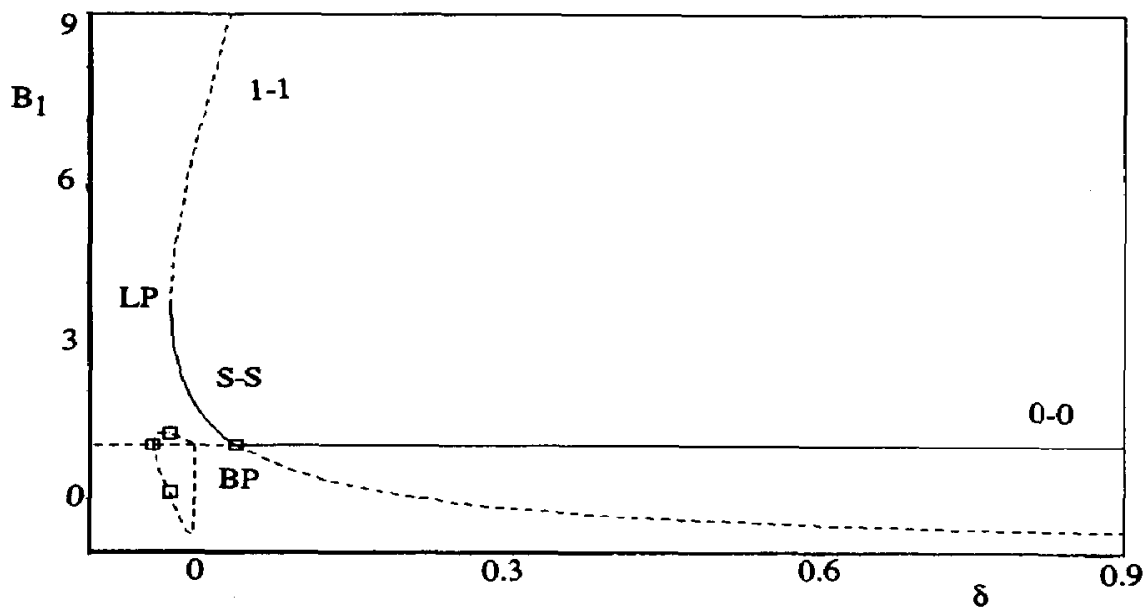

Fig. 5. The branch point of the two virgin states of the AB model in a parameter continuation of $\delta$ for $\mu=0.008$. Whenever $\delta<0.04$ the S-S state is stable, otherwise the $0-0$ state is stable. At negative values of $\delta$ the $S-S$ states connects to the 1-1 state in a limit point. 


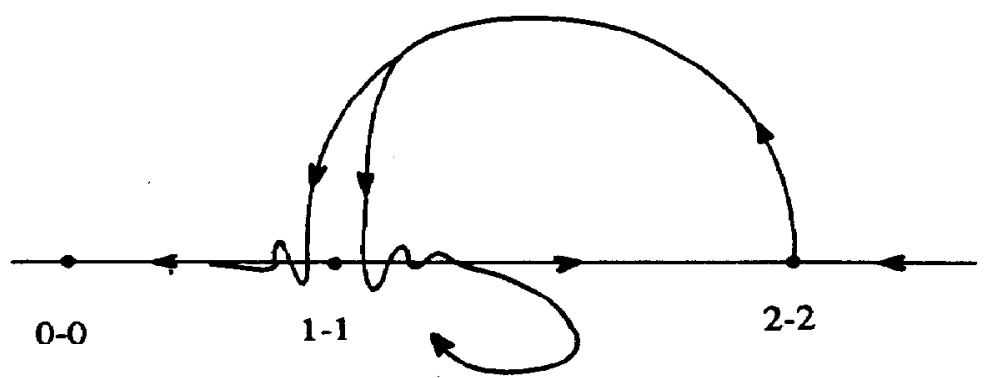

Fig. 6. A schemc of the double heteroclinic connection between the 1-1 and the 2-2 state in the AB model. We have indications for a global bifurcation around $\mu=0.041$.

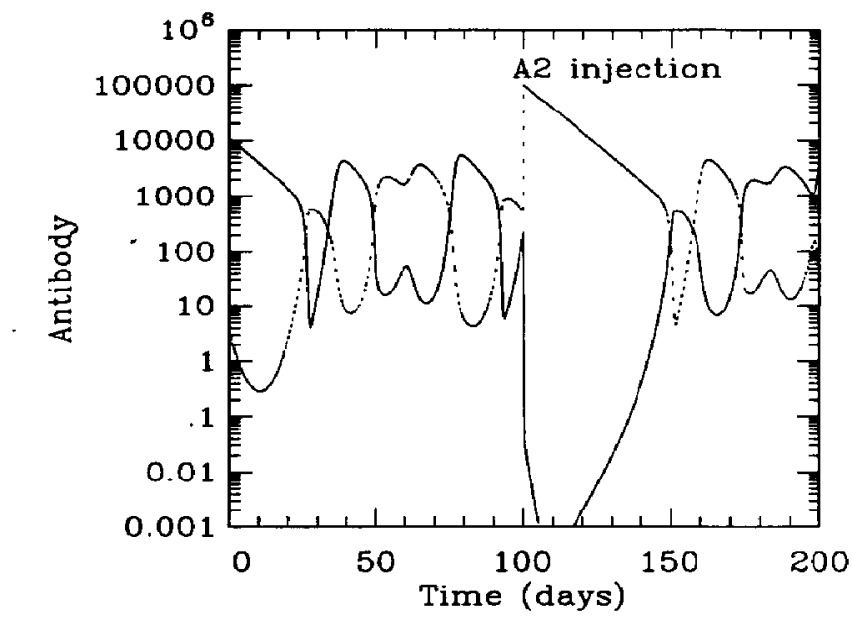

Fig. 7. A chaotic time series of $A_{1}$ and $A_{2}$ populations of the AB model. At day $100 A_{2}$ (dashed line) is set to $10^{5}$. This depresses the oscillations for about 50 d. Parameters: $\mu=0.008, \delta=0.1$.

\section{DISCUSSION}

Cells of the immune system interact with each other and with their environment by chemical means. They express on their surfaces a variety of receptors that detect the presence of antigen and molecules released by other cells (cytokines). The various ligand-receptor interactions signal the cell and singly, or in concert, can lead to modifications of its behavior. Although the biochemistry and kinetics of many ligand rcceptor interactions have been well-studied, there is a need to couple these chemical interactions to the dynamics of the cell population. Here we have begun exploring the consequences of $\mathrm{B}$ cell proliferation and differentiation being controlled via idiotypic interactions. The simplest network model, which contains only two populations interacting through the means of secreted antibody, exhibits a bewildering array of complex dynamic behavior. Using numerical bifurcation techniques we were able to catalog some of the possible behaviors for realistic parameter ranges. We discovered a certain correspondence with recent experimental data that make us believe that chaos and oscillations at the level of single clones may underlie the more regular behavior seen at the level of serum antibody or total $\mathbf{B}$ cell population. 
Idiotypic network theory remains a speculative issue. Previous models have aimed at grasping the essence of the idea and have not paid much attention to the detailed chemistry that is involved in activating $B$ cells by antiidiotypic antibodies. This oversight can influence ones assessment of the models appropriateness. For example, the cycles that we find in the ABC model have a period of about two months, whereas those in the $A B$ model without chemistry have a period of just two weeks. Immunological data are as yet insufficient to decide which of these two periods is most correct. In fact, evidence exists for both (Rodkey and Adler, 1983; Lundkvist et al., 1989).

As the level of quantitation in immunology increases, the approach taken here should prove to be ever more valuable. Examing long term dynamics, and bifurcations with respect to operaling parantelers, is a rnucl stronger tool with which to qualitatively discriminate among models than the simple measurements of transients that immunologists may use. It thus follows that if experiments were performed in vitro, where one may be able to select parameters over a wider range, and in a chemostat environment where steady states may be maintained, we would be able to do a better job at model discrimination, probing, and eventually understanding the immune response.

\section{ACKNOWLEDGEMENTS}

Portions of this work were done under the auspices of the U. S. Department of Energy. I.G.K. also gratefully acknowledges the support of the National Science Foundation through a PYI award, and a Packard Foundation Fellowship.

\section{REFERENCES}

De Boer, R. J. (1983). GRIND: Great integrator differential equations. Bioinformatics Group, Univ. of Utrecht, The Netherlands.

De Boer, R. J. and P. Hogeweg (1989a). Memory but no suppression in low-dimensional symmetric idiotypic network models. Bull. Math. Biol. 51, 223-246.

De Boer, R. J. and P. Hogeweg (1989b). Unreasonable implications of reasonable idiotypic network assumptions. Bull. Math. Biol. 51, 381-408.

Doedel, E. J. (1981). AUTO: a program for the bifurcation analysis of autonomous systems. Cong. Num. 30, 265-285.

Kevrekidis, I. G. and M. S. Jolly (1987) On the use of interactive graphics in the numerical study of chemical dynamics. Paper no. 22c, A.I.Ch.E. Annual Meeting, New York, NY.

Jerne, N. K. (1974). Towards a network theory of the immune system. Ann. Immunol. (Inst. Pasteur) 125 C, 373-389.

Lorenz, E. N. (1963). Deterministic nonperiodic flow, J. Atmos. Sci 20, 130-141.

Lundkvist, I. , A. Coutinho, F. Varela, and D. Holmberg (1989). Evidence for a functional idiotypic network among natural antibodies in normal mice. Proc. Natl. Acad. Sci. USA, 86, 5074-5078.

Perelson, A. S. and C. DeLisi (1980). Receptor clustering on a cell surface. I. Theory of receptor cross-linking by ligands bearing two chemically identical functional groups. Math. Biosciences 48:71-110.

Perelson, A. S. (1984). Some mathematical models of receptor clustering by multivalent ligands. In: Cell Surface Dynamics: Concepts and Models, (A. S. Perelson, C. DeLisi and F. W. Wiegel, Eds.) Marcel Dekker, New York, pp. 223-276.

Perelson, A. S. (1989). Immune network theory. Immunol. Rev., 110, 5-36.

Segel L. A. and A. S. Perelson (1989). Shape space analysis of immune networks. In Cell to Cell Signalling: From Experiments to Theoretical Models, (A. Goldbeter, Ed.), Academic Press, New York, pp. $273-283$.

Rodkey, L.S. and F. L. Adler (1983) Regulation of natural antiallotype antibody responses by idiotypic networkinduced auto-antiidiotypic antibodies. J. Exp. Med. 157, 1920-1931.

Sparrow, C. (1982). The Larenz Equations: Bifurcations, Chnos, and. Strange Attractors. Springer, New-York. Weisbuch, G., R. J. De Boer and A. S. Perelson (1990). Localized memories in idiotypic networks. J. Theor. Biol., in press. 